\section{Delinquentes Verhalten bei ADHS-Patienten}

Deutsche Wissenschaftler haben den Zusammenhang zwischen delinquentem Verhalten und der Aufmerksamkeitsdefizit-Hyperaktivitätsstörung (ADHS) untersucht. Es zeigte sich, dass der Schweregrad der Delikte bei ADHS-Patienten größer ist und das Einstiegsalter in die Kriminalität geringer.

n der Klinik für Forensische Psychiatrie Rostock wurden 67 Patienten des Entziehungsmaßregelvollzugs nach $₫ 64$ StGB auf das Vorliegen einer ADHS untersucht. Für die Diagnose wurde von den beiden Autoren die Langversion der Wender Utah Rating Scale (WURS) sowie der ADHS-SB-Fragebogen eingesetzt. Zur Sicherung der Diagnose wurden die in diesem Screening auffälligen Patienten mithilfe der Connors Adult ADHD (Selbst- und Fremdbeurteilung) Rating Scale untersucht. Zusätzlich fand eine Testung der Intelligenz (HAWIE-R) statt.

Die Prävalenz der ADHS-assoziierten Symptome betrug $65,7 \%$. Gewaltdelikte standen mit $76 \%$ im Vordergrund. Das
Einstiegsalter in die Kriminalität bei schwer von ADHS Betroffenen lag mit 16 Jahren deutlich unter dem Alter von 19 Jahren der nur leicht von ADHS Betroffenen. Die Einteilung in die Untergruppen der ADHS zeigte, dass Patienten mit Typ III (hyperkinetisch/impulsiv) häufiger betroffen waren als Patienten vom vorwiegend unaufmerksamen oder gemischten Typ. Interessanterweise waren aber teilremittierte ADHS-Patienten genauso häufig betroffen und fanden sich signifikant häufiger in der Gruppe der schweren Gewaltdelikte (Mord/Totschlag).

Engel J, Schläfke D. ADHS in einer Entziehungsmaßregel. Nervenheilkunde 2012, 1-2: 42-7
Kommentar: ADHS ist ein Risikofaktor für Delinquenz, das wurde bereits in zahlreichen Studien belegt - auch in Deutschland (Rösler M. et al Eur Arch Psychiatr Clin Neurosci 2004; 254: 365-71). Langzeituntersuchungen von Barkley weisen jedoch darauf hin, dass überwiegend die komorbide Störung des Sozialverhaltens dafür verantwortlich ist, die schon seit Kindheit besteht. Dies wurde in der vorliegenden Untersuchung leider nicht nachgeprüft. Die gestörte Impulssteuerung, die vor allem in der Untergruppe III dominiert, könnte aber ebenfalls zu einer erhöhten Delinquenz vor allem bei nicht geplanten Delikten beitragen. Eine erhöhte Delinquenzrate im Subtyp III der Studie bestätigt dies. Aufgrund der kleinen Fallzahlen sind hierüber aber keine eindeutigen Aussagen zu treffen. Fakt bleibt, dass es sinnvoll ist, Jugendliche im Strafvollzug auf eine ADHS hin zu untersuchen. Bei vorliegender ADHS-Diagnose könnte eine adäquate Therapie den Betroffenen eine bessere Prognose ermöglichen.

\section{Dr. Kirsten Stollhoff}

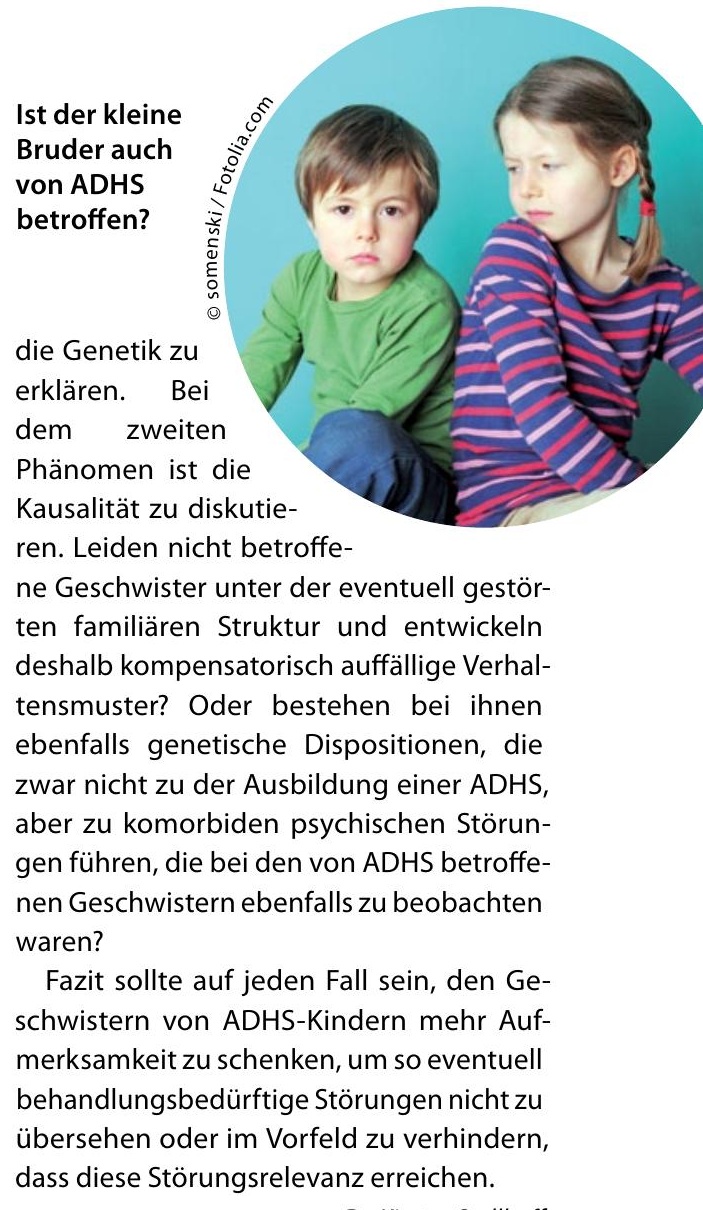

Dr. Kirsten Stollhoff 